\title{
Study of the Effects of Walnut Leaf on Some Blood Biochemical Parameters in Hypercholesterolemic Rats
}

Mehdi Mahmoodi ${ }^{*}$, Hadis Eghbali², Seyed-Mostafa Hosseini-zijoud ${ }^{3}$, Ahmad Pourrashidi $^{4}$, AliReza Mohamadi $^{5}$, Majid Borhani ${ }^{5}$, Gholamhossein Hassanshahi ${ }^{6}$, Mohsen Rezaeian ${ }^{7}$

${ }^{1}$ Professor of Clinical Biochemistry Department of Biochemistry, Faculty of Medicine and Molecular Medicine Research center, University of Medical Sciences, Rafsanjan, Iran

${ }^{2} \mathrm{MSc}$, Department of Biochemistry, Faculty of Medicine, University of Medical Sciences, Rafsanjan, Iran

${ }^{3}$ M.Sc Student of Clinical Biochemistry, Department of Biochemistry, Faculty of Medicine, University of Medical Sciences, Rafsanjan, Iran

${ }^{4}$ General Physician, Department of Biochemistry, Faculty of Medicine, University of Medical Sciences, Rafsanjan, Iran

${ }^{5}$ Molecular Medicine Research Center, University of Medical Sciences, Rafsanjan, Iran

${ }^{6}$ Associate Professor of Hematology, Molecular Medicine Research Center, University of Medical Sciences, Rafsanjan, Iran

${ }^{7}$ Associate Professor of Epidemiology, Department of Social Medicine, Faculty of Medicine, University of Medical Sciences, Rafsanjan, Iran

\section{Abstract}

Hypercholesterolemia is characterized as a major risk factor for cardiovascular diseases (CVD). The use of antioxidants is the most preferred way to overcome hypercholesterolemia complications. Several evidences demonstrated the potential antioxidant properties of walnut leaf, so current study was designed to examine this effect in hypercholesterolemic rats.

Fifty albino rats divided into 5 groups of 8 . Hypercholesterolemic rats (1\% cholesterol in usual diet) received $1 \%$, $2 \%$ and $5 \%$ weight concentration of walnut leaf powder in daily diets. There is two Hypercholesterolemic and normal controls too.

The treatment period was 40 days. The blood samples were collected from eyes after treatment, and biochemical parameters including FBS, triglycerides, cholesterol, low density lipoprotein cholesterol (LDL-C) and high density lipoprotein cholesterol (HDL-C) were assayed.

The current results showed that consumption of walnut leaf decreased cholesterol $(P<0.05), L D L-C$ and triglycerides but increased HDL-C. The consumption of $5 \%$ walnut leaf in hypercholesterolemic diet had most effects.

It can be concluded that walnut leaf has useful effects probably due to its antioxidant properties, on cholesterol and lipid profile in hypercholesterolemic rats and it could be used for reduced CVD risks disease.

Keywords: Hypercholesterolemia; Walnut leaf; Cardiovascular disease

\section{Introduction}

Heart disease is one of the most leading risk factors of death in the United States [1] and is predicted to be the main cause of death worldwide by 2020 [2]. Hypercholesterolemia is a major risk factor for coronary artery disease and thus for myocardial infarction and stroke [3]. High serum LDL-C levels particularly pose a significantly increased risk for heart disease 3, 4 that increase death rate of coronary artery patients.

Hypercholesterolemia is more prevalent among industrials societies [4]. Hypercholesterolemia diets increase cholesterol, LDL-C and triglycerides. On the other hand it reduces LDL-receptor activities in liver. Elevating of serum HDL-C can prevent development of hypercholesterolemia and cardiovascular disease (CVD) [5]. Previous studies showed that antioxidants may reduce the possibility of CVD occurrence due to hypercholesterolemia [5].

The use of antioxidants is the most preferred way to inhibit lipid oxidation [6]. Recently, some negative side effects of the commonly used synthetic antioxidants have been established. Evidences revealed that these compounds may be implicated in many health risks, including cancer and carcinogenesis $[7,8]$.

Therefore, there is a tendency towards the use of plant origin natural antioxidants instead of synthetic antioxidants. Numerous types of natural antioxidants with various activities have been identified but more attentions recently paid to the addition of polyphenols to plants, due to their role as scavengers of free radicals [8].

The antioxidant activity of phenolic compounds in plants is mainly due to their redox properties and chemical structure. This can play important roles in neutralizing free radicals, chelating transitional metals, and quenching singlet and triplet oxygen molecules through delocalizing or decomposing peroxides.

These properties are linked to beneficial health functionality of phenolic antioxidants due to their inhibitory effects against development of many oxidative-stress related diseases, including cardiovascular disease [9].

Walnut (Juglans regia L.) is a valuable crop being the nut very

*Corresponding author: Mehdi Mahmoodi, Department of Biochemistry, Faculty of Medicine and Molecular Medicine Research Center, University of Medical Sciences, Rafsanjan, Iran, Tel: +98 913191 4855; Fax: +98 391522 5209; E-mail: mahmoodies@yahoo.com

Received November 20, 2011; Accepted December 09, 2011; Published December 10, 2011

Citation: Mahmoodi M, Eghbali H, Hosseini zijoud SM, Pourrashidi A, Mohamadi AR, et al. (2011) Study of the Effects of Walnut Leaf on Some Blood Biochemical Parameters in Hypercholesterolemic Rats. Biochem \& Anal Biochem 1:103 doi:10.4172/2161-1009.1000103

Copyright: (c) 2011 Mahmoodi M, et al. This is an open-access article distributed under the terms of the Creative Commons Attribution License, which permits unrestricted use, distribution, and reproduction in any medium, provided the original author and source are credited. 
Citation: Mahmoodi M, Eghbali H, Hosseini zijoud SM, Pourrashidi A, Mohamadi AR, et al. (2011) Study of the Effects of Walnut Leaf on Some Blood Biochemical Parameters in Hypercholesterolemic Rats. Biochem \& Anal Biochem 1:103. doi:10.4172/2161-1009.1000103

Page 2 of 4

popular and largely consumed. Not only dry fruits (nuts) but also other components of walnuts such as green walnuts, shells, kernels, barks, green walnut husks (epicarp) and leaves have been used in both cosmetic and pharmaceutical industries [10]. Different studies demonstrated the potential antioxidant effects of walnut products, especially fruits, leaves and liqueurs which produced by green fruits $[10,11]$

Walnut leaf is rich in antioxidants components like phenolic acids and flavonoids. Caffeoylquinic and caffeoylquinic acid are the major phenolic acids and Juglone (5-hydroxy-1, 4-naphthoquinone), quercetin and its derivatives are main flavonoids found in walnut leaf $[12,13]$.

There are many studies regarding pharmaceutical effects of walnut leaf [14]. A study demonstrated the hypoglycemic effects of walnut leaf in diabetic rats [15].

In other study flavonoids reduced serum glucose [16]. Also according to Namasivayam study, quercetin reduced cholesterol level significantly beside of blood glucose [17].

There are, however, little reports known about hypoglycemic and hypolipidemic effects of walnut leaf [18], a crossover study conducted by Sabate et al., [19] examined the effects of walnut consumption on serum lipid levels in human subjects. The study of favorable effects of walnuts on serum lipid levels in hypercholesterolemic people displays the ability of walnuts to reduce the risk of heart disease.

Furthermore there aren't many studies about hypocholesterolemic effects of walnut leaf, thus, the current study was aimed to examine this effect of walnut leafs in hypercholesterolemic rat models.

\section{Materials and Methods}

Walnut leaf powder was mixed with usual food in 1\%,2\% and 5\% weight concentration. Then was prepared as pellet and exposed to rats. Normal food for fifth group was available.

Forty albino rats (mean weight, 250-300g) were divided into following 5 groups with 8 animals in each group:

First group received $1 \%$ cholesterol and $1 \%$ walnut leaf powder in daily diets.

Second group received $1 \%$ cholesterol and $2 \%$ walnut leaf powder in daily diets.

Third group received $1 \%$ cholesterol and 5\% walnut leaf powder in daily diets.

Forth group received $1 \%$ cholesterol in daily diet (Hypercholesterolemic control).

Fifth group received normal daily diet (normal control).

The amount of consumed food measured weekly. The treatment period was 40 days and the end of treatment period, blood samples were collected from eyes and biochemical parameters including FBS, triglycerides, cholesterol, LDL-C and HDL-C were assayed.

Data were analyzed by SPSS software and using ANOVA test for comparing results among all groups. A $\mathrm{P}<0.05$ was considered as significant.

Data were analyzed by SPSS software and using statistical tests, ANOVA and paired t-test for comparing results among all groups. A $\mathrm{P}<0.05$ was considered as significant.

\section{Results}

In the current study we tried to assess the impact of walnut leaf powder on lipid profile in hypercholesterolemic rats following 40 days taking walnut leaf powder in daily diets.

Cholesterol consumption increased the levels of triglycerides, cholesterol and LDL-C but decreased HDL-C in forth group (Figure $1,2,3,4)$.

As shown in figure-1, walnut leaf powder in daily diets reduced the triglycerides level in hypercholesterolemic rats while none of them reach to normal level, only the third group that received $5 \%$ concentration of walnut leaf powder in daily diets had significant effect $(\mathrm{P}<0.05)$.

As it is obviously displayed in figure 2 and our statistical data analysis showed treatment of animals with walnut leaf powder in daily diets significantly decreased the level of cholesterol in hypercholesterolemic rats compare to hypercholesterolemic control $(\mathrm{P}<0.05)$.

Finding of the current study also indicated a slightly decrease in

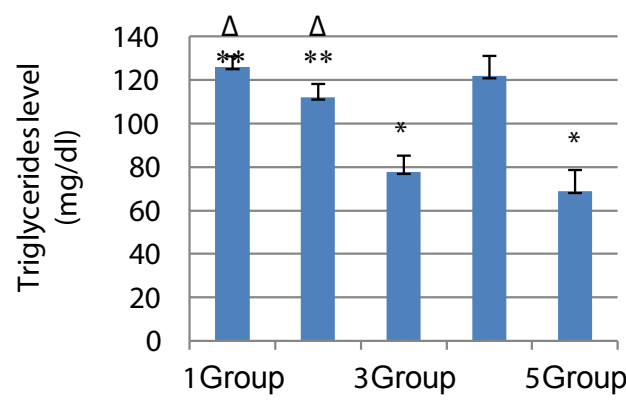

Figure 1: The effect of different concentration of walnut leaf on triglycerides level $(\mathrm{mg} / \mathrm{dl})(\mathrm{P}<0.05)$.

Group1: received $1 \%$ cholesterol and $1 \%$ walnut leaf powder in daily diets. Group2: received $1 \%$ cholesterol and $2 \%$ walnut leaf powder in daily diets. Group3: received $1 \%$ cholesterol and $5 \%$ walnut leaf powder in daily diets. Group4: received 1\% cholesterol in daily diet (Hypercholesterolemic control).

Group5: received normal daily diet (normal control).

* Significant differences with group 4.

** Significant differences with group 5 .

$\Delta$ Significant differences with group 3 .

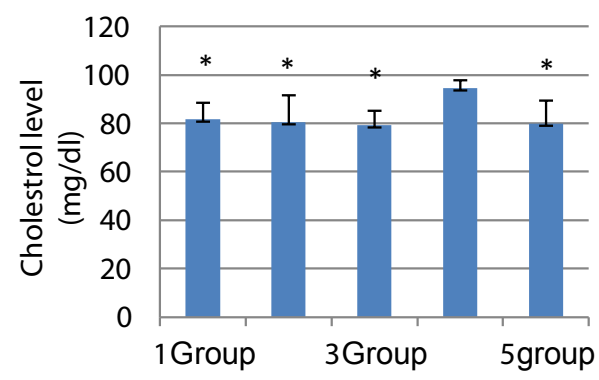

Figure 2: The effect of different concentration of walnut leaf on cholesterol level $(\mathrm{mg} / \mathrm{dl})(\mathrm{P}<0.05)$

Group1: received $1 \%$ cholesterol and $1 \%$ walnut leaf powder in daily diets. Group2: received $1 \%$ cholesterol and $2 \%$ walnut leaf powder in daily diets. Group3: received $1 \%$ cholesterol and $5 \%$ walnut leaf powder in daily diets. Group4: received 1\% cholesterol in daily diet (Hypercholesterolemic control). Group5: received normal daily diet (normal control).

* Significant differences with group 4. 
LDL-C level hypercholesterolemic rats which received walnut leaf powder in daily diets $(\mathrm{P}<0.05)$ (Figure 3$)$.

Treatment of animals with walnut leaf powder in daily diets also increase the HDL-Clevel in treated rats compare to hypercholesterolemic control group $(\mathrm{P}<0.05)$ (Figure 4$)$.

As illustrated in figure 5 hypercholesterolemic diet for 40 days increase FBS while walnut leaf powder in daily diets gently reduce it in dose dependent manner.

\section{Discussion and Conclusions}

Findings of the current study showed that walnut leaf (significantly) reduced cholesterol and LDL where increased HDL-V compares to hypercholesterolemic group.

Treatment with 5\% walnut leaf in hypercholesterolemic diet had most effects on lipid profile and also decreases FBS level in hypercholesterolemic rats.

Walnut leaf contains important reagents such as phenolic acids

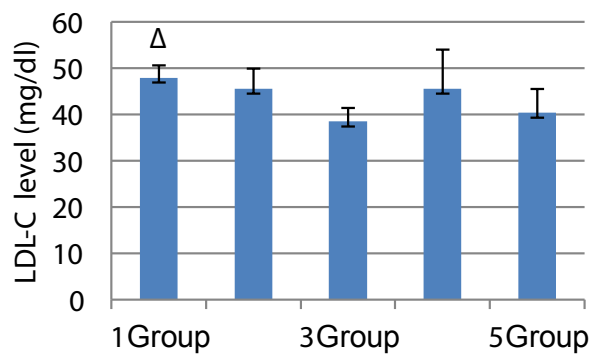

Figure 3: The effect of different concentration of walnut leaf on LDL-C leve (mg/dl) $(\mathrm{P}<0.05)$.

Group 1: received 1\% cholesterol and $1 \%$ walnut leaf powder in daily diets. Group2: received $1 \%$ cholesterol and $2 \%$ walnut leaf powder in daily diets. Group3: received $1 \%$ cholesterol and $5 \%$ walnut leaf powder in daily diets. Group4: received 1\% cholesterol in daily diet (Hypercholesterolemic control). Group5: received normal daily diet (normal control).

$\Delta$ Significant differences with group 3

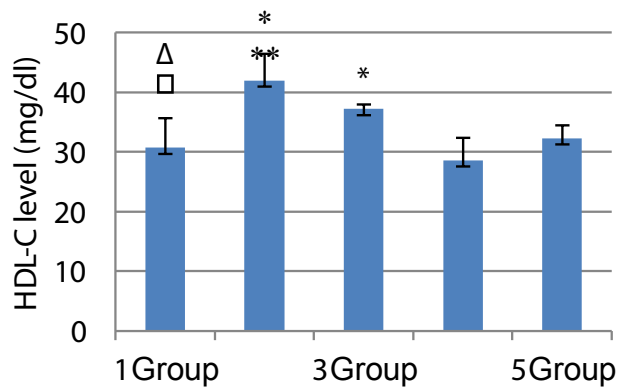

Figure 4: The effect of different concentration of walnut leaf on HDL-C level $(\mathrm{mg} / \mathrm{dl})(\mathrm{P}<0.05)$.

Group 1: received 1\% cholesterol and $1 \%$ walnut leaf powder in daily diets. Group2: received $1 \%$ cholesterol and $2 \%$ walnut leaf powder in daily diets. Group3: received $1 \%$ cholesterol and $5 \%$ walnut leaf powder in daily diets. Group4: received $1 \%$ cholesterol in daily diet (Hypercholesterolemic control). Group5: received normal daily diet (normal control).

* Significant differences with group 4

** Significant differences with group 5

$\Delta$ Significant differences with group 3 .

$\square$ Significant differences with group 2 .

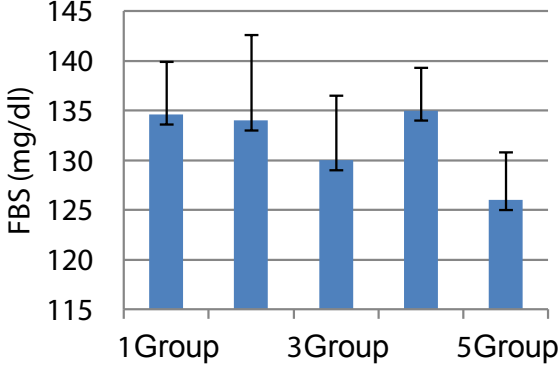

Figure 5: The effect of different concentration of walnut leaf on FBS level (mg/dl).

Group1: received 1\% cholesterol and 1\% walnut leaf powder in daily diets.

Group2: received $1 \%$ cholesterol and $2 \%$ walnut leaf powder in daily diets.

Group3: received $1 \%$ cholesterol and $5 \%$ walnut leaf powder in daily diets.

Group4: received 1\% cholesterol in daily diet (Hypercholesterolemic control).

Group5: received normal daily diet (normal control).

and flavonoids and other vital elements including $\mathrm{Ca}, \mathrm{K}, \mathrm{Mg}, \mathrm{Br}$ and carotene. The possible mechanisms by which walnut leaf may improve lipid profiles may include the effects of these compounds, fiber, micronutrients such as vitamin $\mathrm{E}$ and $\mathrm{C}$, folic acid, copper, magnesium, plant protein (e.g.,arginine), plant sterols, and phenolic components [20]. Fiber in walnut leaf can interfere with lipid and cholesterol intake and metabolism, thus, has useful effect.

Current finding confirmed the positive effect of walnut leaf on FBS level in hypercholesterolemic rats. In a study Divband et al., reported that triglycerides, LDL-C and FBS were reduced in diabetic rats that treated with walnut leaf extracts that are consistent with our results. They also showed that HDL-C was increased by walnut leaf treatment [21].

According to previous studies, walnut leaf also consists of remarkably antioxidant components. These hypoglycemic and hypolipidemic effects have been found in current study may related to its antioxidant properties [22]. Flavonoids are major antioxidants present in walnut leaf [22].

Flavonoids may be induced glycogenesis and inhibit $\mathrm{K}$ channel in pancreas's beta cells and regulate sugar intake from intestine [23]. It also may distribute lipid profile properly and prevent lipid and cholesterol accommodation [23].

A study on diabetic rats demonstrated the reducing effects of walnut leaf ethanolic extract on triglycerides and cholesterol that again is in agreement with our results [24]. In other study, Chlorogenic acid from walnut leaf indirectly interferes in cholesterol synthesis pathway and inhibit HMGcoA reductase enzyme, reduce cholesterol production, also increase its bile secretions [17].

There is other possible mechanism, walnut leaf is good sources of unsaturated fatty acids [monounsaturated fatty acids (MUFA) and PUFA] known for their favorable affects on blood lipids that reduce total cholesterol and LDL-C $[25,26]$.

Current results and previous studies indicate the hypocholestremic properties of walnut leaf. So, based on the results of this investigation it can be concluded that walnut leaf has useful effects on cholesterol and lipid profile in hypercholesterolemic rats and may be it can be used for reducing of CVD risks. 
Citation: Mahmoodi M, Eghbali H, Hosseini zijoud SM, Pourrashidi A, Mohamadi AR, et al. (2011) Study of the Effects of Walnut Leaf on Some Blood Biochemical Parameters in Hypercholesterolemic Rats. Biochem \& Anal Biochem 1:103. doi:10.4172/2161-1009.1000103

\section{References}

1. Anonymous (2003) Statistical Facts Sheet: Leading Causes of Death. American Heart Association: Nutrition Bytes 9: 6.

2. Murray CJL, Lopez AL (1996) the Global Burden of Disease.

3. Anonymous (2002). Atherosclerosis. American Heart Association.

4. Fraser GE (1999) Nut consumption, lipids, and risk of a coronary event. Clin Cardiol 22: 11-15.

5. Khayyat noori M, Jafari R (2006) the effect of vitamin C complementary on cholesterol level in hypercholesteromic rats. Feiz J 10: 37-40.

6. Erkan N, Ayranci G, Ayranci E (2009) A kinetic study of oxidation development in sunflower oil under microwave heating: Effect of natural antioxidants. Food Res Int 42: 1171-1177.

7. Assimopoulou AN, Zlatanos SN, Papageorgiou VP (2005) Antioxidant activity of natural resins and bioactive triterpenes in oil substrates. Food Chem 92 721-727.

8. Zhang Y, Yang L, Zu Y, Chen X, Wang F, et al. (2010) Oxidative stability of sunflower oil supplemented with carnosic acid compared with synthetic antioxidants during accelerated storage. Food Chem 118: 656-662.

9. Oliveira I, Sousa A, Ferreira IC, Bento A, Estevinho L, et al (2008) Tota phenols, antioxidant potential and antimicrobial activity of walnut (Juglans regia L.) green husks. Food Chem Toxicol 46: 2326-2331.

10. Stampar F, Solar A, Hudina M, Veberic R, Colaric M (2006) Traditional walnut liqueur-cocktail of phenolics. Food Chem 95: 627-631.

11. Pereira JA, Oliveira I, Sousa A, Ferreira IC, Bento A, et al (2008) Bioactive properties and chemical composition of six walnut (Juglans regia L.) cultivars. Food Chem Toxicol 46: 2103-2111.

12. Amaral JS, Seabra RM, Andrade PB (2004) Phenolic profile in the quality control of walnut (Juglans regia L.) leaves. Food Chemistry 88: 373-379.

13. Fukuda T, Ito H, Yoshida T (2003) Antioxidative polyphenols from walnuts (Juglans regia L.). Phytochemistry 63: 795-801.

14. Kaumar S, Harkonen PL, Arora S, Kaur M (2003) Studies on correlation of antimutagenic and antiproliferative activities of Juglans regia L. J Environ Pathol Toxicol Oncol 22: 59-67.

15. Jelodar g, nazifi s (2000) The effects of pomegranate, coindiar and walnut lea on some Blood Biochemical parameters in diabetic rats. Shahid sadooqi yazd J 1: 77-82.

16. Vaya J, Aviram M (2002) Nutritional antioxidants: mechanism of action, analyses of activities and medical applications. Curr Med Chem Immuno Endocr Metab Agents 1: 99-117.

17. Namasivayam N (2002) Hypolipidemic effect of Cuminum cyminum L .on alloxan-induced diabetic rats. Pharmacol Res 46: 251-255

18. Fathiazad F, Garjani A, Motavallian naini A (2006) Study of hypoglycemic activity of the hydroalcholic extract of Juglans regia in normal and diabetic rats. Pharmaceutical Sciences 2: 13-17.

19. Sabate J, Fraser GE, Burke K, Knutsen SF, Bennett H, et al (1993) Effects of walnuts on serum lipid levels and blood pressure in normal men. $\mathrm{N}$ Engl $\mathrm{J}$ Med 328: 603-607.

20. Kris-Etherton PM, Zhao G, Binkoski AE, Coval SM , Etherton TD (2001) The effects of nuts on coronary heart disease risk. Nutr Rev 59: 103-111.

21. Divband K, Komeili GR, Saeidi-Neek F (2010) Effects of Walnut leaves aqueous extract on blood sugar and serum lipids in diabetic rats. Journal of Birjand University of Medical Sciences 17: 11-18.

22. Pereira JA, Oliveira I, Sousa A, Valenta P, Andrade PB, et al. (2007) Walnut (Juglans regia L.) leaves: Phenolic compounds, antibacterial activity and antioxidant potential of different cultivars. Food Chem Toxicol 45: 2287-2295.

23. Almario RU, Vonghavaravat V, Wong R, Kasim-Karakas SE (2001) Effects of walnut consumption on plasma fatty acids and lipoproteins in combined hyperlipidemia. Am J Clin Nutr 74: 72-79.

24. Jelodar g, nazifi s (1999) The effects of walnut leaf on some Blood Biochemica parameters in diabetic rats. Shahid beheshti $\mathrm{J} 2: 18-25$.

25. Mensink RP, Katan MB (1992) Effect of dietary fatty acids on serum lipids and lipoproteins. A meta-analysis of 27 trials. Arterioscler Thromb 12: 911-919.

26. Hegsted DM, Ausman LM, Johnson JA, Dallal GE (1993) Dietary fat and serum lipids: an evaluation of the experimental data. Am J Clin Nutr 57: 875-883. 